\title{
Dante Gatteschi: on the Occasion of His 75th Birthday
}

\section{Lorenzo Sorace $^{1} \cdot$ Maria Fittipaldi $^{2} \cdot$ Donatella Carbonera $^{3}$}

Published online: 13 October 2020

(c) Springer-Verlag GmbH Austria, part of Springer Nature 2020

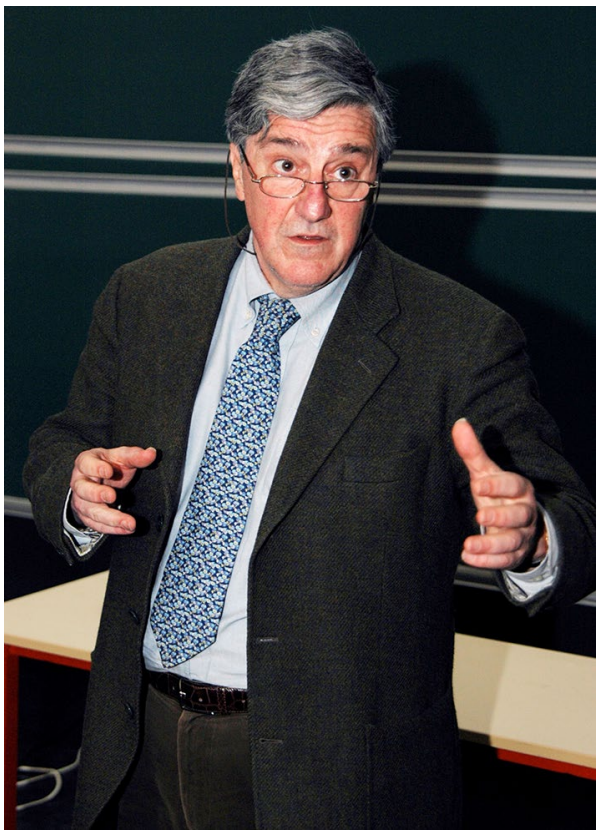

It is with great pleasure that we present this Special Issue of Applied Magnetic Resonance to celebrate the 75th birthday of Prof. Dante Gatteschi (Emeritus Professor at

Lorenzo Sorace

lorenzo.sorace@unifi.it

1 Dipartimento di Chimica "U. Schiff" and UdR INSTM, University of Florence, Via della Lastruccia 3, 50019 Sesto Fiorentino, Italy

2 Dipartimento di Fisica and UdR INSTM, University of Florence, via G. Sansone 1, 50019 Sesto Fiorentino, Italy

3 Department of Chemical Sciences, University of Padova, via Marzolo 1, Padova, Italy 
the University of Florence, Italy). Dante Gatteschi was born in Florence on October 27th, 1945. He graduated in Chemistry at the University of Florence in 1969, a time when in Italy there was no PhD program, under the guidance of Prof. Luigi Sacconi, founder of the School of Inorganic Chemistry in Florence. Soon after graduation, Dante Gatteschi became Assistant Professor at the University of Florence, where he spent all his academic career, becoming Professor of General and Inorganic Chemistry in 1980. He is Emeritus Professor of University of Florence since 2015.

The research of Dante Gatteschi early focused on the use of ligand field theory for the interpretation of the spectro-magnetic properties of low symmetry transition metal compounds, a hot topic in the '70s. The focus on copper(II) compounds undergoing Jahn-Teller distortion led him quite naturally to the application of EPR spectroscopy to understanding the static and dynamic structures of transition metal ions. The fundamental work performed on low symmetry $\mathrm{Co}$ (II) complexes still remains nowadays as unmissable. The natural extension of these investigations was towards systems in which metal ions are magnetically coupled, first in pairs, and later in more complex structures, such as oligonuclear systems and molecular chains. Again, the use of EPR in this area has been extensive, leading to the book he co-authored with Alessandro Bencini on "EPR of Exchange Coupled Systems", which is a fundamental reference in the field. The development of the molecular magnetism research field lead Prof. Gatteschi to make large use of nitronyl nitroxide radicals, either as purely organic ferromagnets or as building blocks for more complex architectures: here EPR played again a pivotal role in determining the magnetic behaviour of these systems. A major breakthrough came in the '90s, when the use of High Field High-Frequency EPR facility in Grenoble allowed Dante and his coworkers to elucidate the reasons which lead a dodecanuclear mixed-valence Manganese cluster to behave as a tiny magnet at very low temperature and to show unequivocal signatures of quantum tunnelling of the magnetization. This gave birth to the field of Single Molecule Magnetism, and provided much impulse to the development of HFEPR in Europe and all around the world. With the new millennium, the interest of Dante Gatteschi in EPR shifted towards the characterization of magnetic nanoparticles of progressively smaller size, and the way a unified approach for the treatment with high nuclearity spin cluster can be achieved.

In his career, Dante Gatteschi has coauthored more than 530 publications in scientific journals and he is coauthor/coeditor of 6 books.

These impressive scientific results were accompanied by an intense activity in promoting the development of appropriate conditions for successful scientific research at a national and international level. With this aim, at the beginning of the '90s he was one of the founders of National Institute of Materials Science and Technology (INSTM) which brought Italian Universities and research centers working together in the field of materials science. Following the same thread, he promoted the collaboration of a network of European laboratories within many EU Framework Projects substantially contributing to the development of the European school of molecular magnetism. All these results were obtained by developing intense human relationships, as proved by the enthusiasm of contributors to this Special Issue.

Dante Gatteschi is a member of several scientific organizations: Member of the Accademia Nazionale delle Scienze detta dei Quaranta, Member of the Accademia 
Nazionale dei Lincei, Member of Deutsche Akademie der Naturforschung Leopoldina, Member of the Akademia Europea, Fellow of the International EPR Society. For his scientific achievements in the field of magnetism and magnetic resonance of nanosystems he has been awarded several national and international prizes, of which we mention a few in the following: Nasini Award of the Italian Chemical Society, 1979; Magnetic Resonance Award of the Italian Chemical Society, 1993; Bruker Prize for EPR Spectroscopy, 2000; Agilent Technologies Europhysics Prize, 2002; Franco-Italian Prize of the French and Italian Chemical Society, 2007; Cannizzaro Medal, Italian Chemical Society, 2014; International Zavoisky Award, 2015. He further received several honoris causa degrees: Laurea h.c. in Material Science, University of Cagliari, Italy, 2005; Doctorate h.c., University of Bucharest, Romania, 2011; Doctorate h.c. University of Stuttgart, Germany, 2013.

We hope that these 23 papers, containing both original contributions and reviews from friends and colleagues all around the world, will be of great interest to many senior and junior scientists and will provide a glimpse of the different fields touched upon by Dante in his longstanding career. The selected contributions range from the determination of single-ion anisotropy in molecular or bio-inorganic compounds, making ample use of ligand field arguments to rationalize the observed results, to the characterization of organic spin chains and high spin organic polymers; from the application of EPR to solve problems in fields as different as catalysis and mineralogy to application of advanced techniques and instrumental developments; theoretical papers and perspectively new fields of research are also presented. Finally, original works on solid-state NMR of molecular magnets, much used by Gatteschi in collaboration with Prof. Borsa's group, are also included. The number and the quality of these cotributions are even more significant, and indicative of the importance of Dante's contribution in the field of Magnetic Resonance if one considers that the invitation letter for this issue was sent out just a week before WHO declared COVID-19 a pandemic and lockdowns around the world followed.

For these reasons, we heartily thank all the contributors and we are grateful to Prof. Kev Salikhov and Dr. Laila Mosina for their support and encouragement in all phases of the preparation of this special issue. In much the same way, our thanks go to the referees, who provided constructive criticisms thereby contributing to the improvement of the submitted manuscripts.

\author{
Lorenzo Sorace (Lead Guest Editor) \\ Maria Fittipaldi (Co-Editor) \\ Donatella Carbonera (Co-Editor)
}

Publisher's Note Springer Nature remains neutral with regard to jurisdictional claims in published maps and institutional affiliations. 